\section{IMS}

médecine sciences $1988 ; 8: 47+-175$

\section{Jean-Pierre Changeux}

\section{REEFÉRENCES}

1. Changeux JP. Molécule et Mémoire. Bedou : Gourdon, 1988.

2. Hökfelt T, Holets VR, Staines W, et al. Coexistence of neuronal messengers. An overview. Progr Brain Res $1986 ; 68$ : 33-70.

3. Changeux JP, Giraudat J, Dennis M. The acetylcholine receptor: molecular architecture of a ligand-regulated ion channel. Trends Pharmacol Sci $1987 ; 8$ : 459-65.

4. Lindstrom J, Schoepfer R, Whiting P. Molecular studies of the neuronal nicotinic acetylcholine receptor family. Mol Neurobiol $1987 ; 1: 281-337$

5. Jimenez F. Genetic control of neuronal determination in insects. Trends Neurosci 1988: $11: 378-80$.

6. Breakefield XO, Geller AI. Gene transfer into the nervous system. Mol Neurobiol 1987 $1: 339-71$.

7. Sejnowski T J, Rosenberg CR. NET talk a parallel network that learns to read aloud. Technical report JHU/EECS-86/01. Baltimore: MD Johns Hopkins University, 1986.

\section{ADRESSE}

J.-P. Changeux: Professeur à l'Institut Pasteur et au Collège de France. Institut Pasteur, 28,

rue du Dr Roux, 75724 Paris Cedex 15, France

\title{
DE LA MOLÉCULE AU LANGAGE : LA BIOLOGIE DU CERVEAU EN PLEIN ESSOR
}

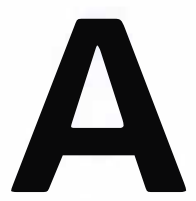

u cours des deux dernières décennies, les sciences du système nerveux se sont trouvées au centre d'une révolution qui paraît bien silencieuse si on la compare au tapage médiatique de la conquête de l'espace ou de la maîtrise de l'énergie. Il s'agit, cependant, de la plus importante conquête que l'homme puisse jamais faire : celle de son propre cerveau. Cette révolution tient à deux causes principales: la première est philosophique, voire idéologique. Le clivage entre le corps et l'esprit n'est plus de mise. Bien au contraire, le sommaire de ce numéro de médecine/sciences le démontre. L'approche multidisciplinaire des fonctions supérieures du cerveau a l'objectif opposé : assigner à chaque processus psychologique une organisation neurale aussi délimitée et précise que possible. Mieux encore, il est désormais envisageable de compléter la démarche de «réduction » vers le physicochimique par l'entreprise plus difficile et exigeante de «reconstruction » du niveau moléculaire au niveau cellulaire, du neurone à l'assemblée de neurones, jusqu'au niveau le plus élevé qui soit: celui de la raison [1].

L'autre cause de la révolution des neurosciences est méthodologique. Il y a quelques années encore, l'anatomie du cerveau constituait un monde bien distinct de la physiologie. L'une se cantonnait dans l'examen de coupes colorées, l'autre de la mesure de paramètres électriques. La psychologie était enseignée avec la philosophie à la faculté des lettres et, plus lourd encore de conséquences, les maladies du système nerveux étaient réparties en deux ensembles arbitrairement étiquetés de neurologie et psychiatrie. L'article de M.F. Chesselet sur la chorée de Huntington souligne fort à propos l'illusoire de cette distinction. Il illustre l'éclatement des disciplines traditionnelles avec l'entrée en force de nouvelles techniques d'étude du cerveau, et plus encore, par leur emploi concerté avec les méthodes classiques. L'alliance de l'épidémiologie et de la génétique moléculaire, de l'histologie et de la chimie des neurotransmetteurs, de l'anatomie et des sciences cognitives, permet désormais de cerner cette maladie dégénérative et d'ouvrir des voies de recherches nouvelles vers des traitements possibles. La biologie du cerveau peut désormais poser des questions théoriques de la plus grande portée philosophique en toute liberté et nourrir l'ambition d'y répondre de manière objective.

L'essor contemporain des neurosciences se développe suivant trois axes principaux: la biologie moléculaire, la biologie du développement, les fonctions supérieures du cerveau. Les communications entre cellules nerveuses se réduisent essentiellement au transfert de signaux chimiques, neurotransmetteurs ou hormones, d'une cellule à l'autre. Comme le décrit H. Korn, dans son article sur la libération des neurotransmetteurs dans le système nerveux central, leur libération s'effectue de manière discrète par paquets d'environ 10000 molécules de neurotransmetteurs avec une probabilité inférieure à l lorsque la terminaison est envahie par un influx nerveux. Cette probabilité est comprise entre 0,2 et 0,6 , ce qui introduit un élément stochastique fondamental dans le transfert de l'information dans le réseau nerveux.

Le répertoire des signaux de communication s'est considérablement accru, ces dernières années, par l'identification de nouveaux neuropeptides et par 
le clonage et la séquence de leurs gènes. Il était admis depuis le temps de Sir Henry Dale qu'un neurone ne libère qu'un seul et même neurotransmetteur par toutes ses synapses. On sait aujourd'hui qu'il n'en est pas ainsi et que plusieurs neurotransmetteurs et neuropeptides peuvent être synthétisés et libérés par un même neurone [2]. L'isolement et le déchiffrage de la structure primaire des récepteurs de ces signaux chimiques (acétylcholine, GABA, glycine... et plusieurs peptides) a fait un bond en avant considérable principalement par l'apport de l'ingéniérie génétique. Les incidences des résultats déjà obtenus sur la pharmacologie du système nerveux sont multiples. D'une part, il a été découvert qu'une même molécule de récepteur peut reconnaître, en plus du neurotransmetteur et de ses analogues, des effecteurs dits «allostériques » [3] dont la chimie est entièrement différente, et qui se lient à un site topographiquement distinct du site récepteur (l'exemple le plus connu est celui de l'activation du récepteur du GABA par les benzodiazépines). D'autre part, des «familles » de gènes homologues [4] codent pour des formes moléculaires distinctes du récepteur d'un même neurotransmetteur présentant chacune une spécificité pharmacologique différente; mieux, ces diverses isoformes s'expriment de manière différente dans différentes régions du cerveau, ouvrant des perspectives très nouvelles en psychopharmacologie. Enfin, l'efficacité de la réponse d'un récepteur, comme celle de la libération d'un neurotransmetteur, peut être modifiée par l'activité qui circule dans le réseau et l'on peut spéculer que de tels processus participent à la mise en place des traces de mémoire.

Le développement du cerveau pose un problème théorique majeur. Comment une organisation aussi complexe se construit-elle à partir de l'œuf et d'un stock de gènes somme toute relativement restreint (moins de 100000 gènes de structure) qui ne diffère que de manière extrêmement modeste entre le singe et l'homme? La génétique moléculaire $a$, là encore, conduit au succès avec l'isolement et l'identification, principale- ment chez la drosophile (mais également chez les vertébrés) de gènes qui déterminent les coordonnées cartésiennes du corps de l'animal, sa segmentation, l'identité de ses segments, et qui s'expriment, pour certains, de manière privilégiée au niveau du système nerveux [5]. D'autres gènes commandent la différenciation de l'ectoderme embryonnaire en tissus nerveux, la ségrégation de certaines catégories de neurones, leur migration et la formation de synapses interneuronales définies. Les protéines de «surface», interviennent de manière essentielle en tant que protéines d'adhésion au cours des multiples étapes successives de la neurogenèse et de la synaptogenèse, ainsi que les facteurs de croissance du type du NGF (nerve growth factor) dont le catalogue s'accroît sans cesse. Il faut s'attendre à ce que beaucoup de maladies dégénératives du système nerveux résultent de la perturbation de ces gènes de développement et/ou des produits de leur expression. La stratégie «de la maladie - au gène » et «du gène - à la protéine» employée avec succès avec la dystrophie de Duchenne (encore en cours avec la chorée de Huntington) devrait pouvoir être étendue à celles de ces maladies dont la composante héréditaire est démontrée. Des thérapies géniques par transfert de gènes [6] peuvent désormais être prises en considération sérieusement.

Une autre méthode d'avenir est la transplantation de tissu ou de cellules nerveuses dont C. Sotelo brosse un portrait prometteur. L'implantation d'un greffon de cervelet normal dans le cervelet d'une souris mutante dépourvue de cellules de Purkinje permet l'intégration de nouvelles cellules de Purkinje dans le réseau synaptique de l'hôte et donc, de réparer, d'une manière significative bien qu'encore modeste, la lésion génique.

Le troisième secteur vers lequel évoluent les neurosciences est fascinant: comprendre les fonctions supérieures du cerveau de l'homme et tenter de les reproduire artificiellement avec une machine. L'ambition est immense, les résultats encore minces mais encourageants. L'approche la plus directe consiste à suivre chez l'homme les conséquences de lésions accidentelles circonscrites et à identifier les territoires et voies nerveuses engagées dans une performance comportementale définie et, dans la mesure du possible, de développer un modèle expérimental chez le singe. Avec l'animal, le débrouillage de la connectivité et l'enregistrement des activités mises en jeu par le codage des mouvements volontaires ont été réal isés avec succès, comme le montre l'article de $\mathbf{M}$. Jeannerod et J.-P. Joseph. Les auteurs révèlent l'existence d'une spécialisation hémisphérique dans les contrôles cognitifs de l'acte moteur et mettent en relief l'intervention du lobe frontal dans les fonctions dites «supérieures» telle que l'intention, la représentation du but à atteindre, l'analyse du contexte dans lequel se déroule l'action, la décision d'agir... L'image que l'on se faisait depuis Lashley du cortex cérébral comme un manteau uniforme et peu réactif doit être abandonnée au profit d'une parcellisation et d'une spécialisation de plus en plus poussée de celui-ci. C'est ce que démontrent avec élégance $\mathrm{S}$. Valdois et $\mathrm{Y}$. Joanette dans leur analyse des trouble du langage chez l'homme, qui s'enrichit des techniques modernes de l'imagerie médicale, comme la caméra à positon. La résolution dynamique est telle que l'on observe directement des territoires distincts du cortex cérébral «allumés» par les composantes visuelles et sémantiques engagées dans la lecture d'un mot. De là à construire des machines à lire et à traduire ou des ordinateurs qui inventent des stratégies de résolution de problèmes! La machine qui apprend à lire à voix haute existe [7]. Mais elle est encore très fruste: elle a besoin d'un «maître » qui corrige sans cesse les fautes lors de l'apprentissage et surtout elle ne «comprend » rien à ce qu'elle lit. Il reste encore beaucoup de chemin à parcourir avant de construire une machine qui écrive une tragédie de Racine! Mais les recherches concertées des mathématiciens et des neurobiologistes, des physiciens et des psychologues devraient faire avancer rapidement le projet de machines qui soient réellement «intelligentes » 